
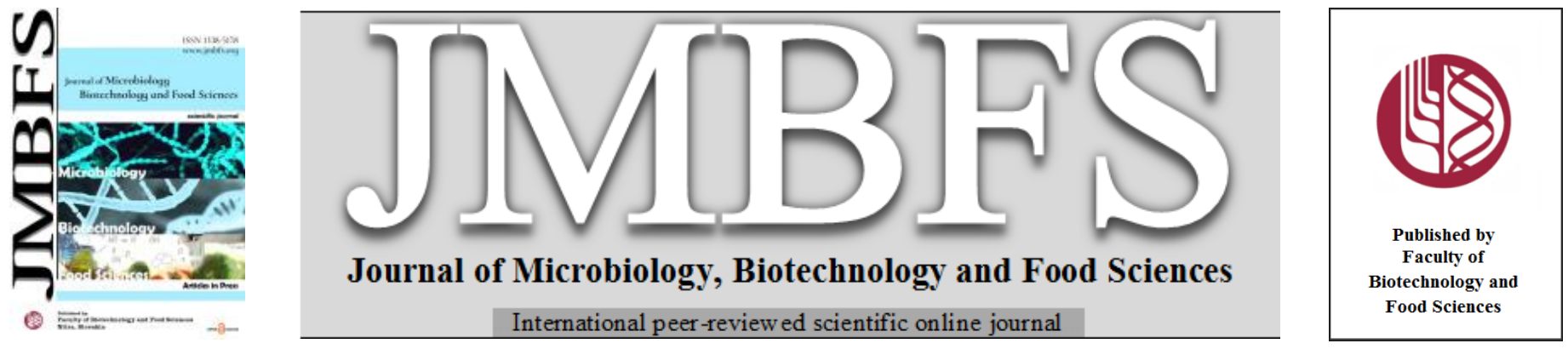

\title{
CHEMICAL CHARACTERIZATION OF VOLATILE EXTRACT OF ARTEMISIA HERBA-ALBA AND STUDY OF ITS ANTIOXIDANT, ANTIMICROBIAL AND ANTIFUNGAL ACTIVITIES AND ITS INHIBITIONORY EFFECT ON CORROSION OF ALUMINUM IN HYDROGEN CHLORIDE SOLUTION
}

\author{
Yamen Ouchelli $^{1}$, Nacera Dahmani-Hamzaoui ${ }^{1,2 *}$ Yassine Addi $^{3}$, Nacer Hechiche ${ }^{4}$, Aoumar Baaliouamer ${ }^{2}$
}

\author{
Address(es): \\ ${ }^{1}$ Université des Sciences et de la Technologie Houari Boumediene, Faculté de Chimie, Laboratoire d'Analyse Organique Fonctionnelle, BP 32 Al-Alia, Bab-Ezzouar \\ 16111, Alger, Algeria. 00213670282264. \\ ${ }^{2}$ Université Mouloud Mammeri, Faculté des Sciences, Département de Chimie, Tizi-Ouzou, Algeria. 00213670282264. \\ ${ }^{3}$ Université des Sciences et de la Technologie Houari Boumediene, Faculté de Chimie, Laboratoire de Chimie des Matériaux, BP 32 Al-Alia, Bab-Ezzouar 16111, Alger, \\ Algeria.
}

${ }^{4}$ Université Mouloud Mammeri, Faculté des Sciences, Département de Chimie, Laboratoire de Physique et Chimie des Matériaux, Tizi Ouzou, Algeria.

*Corresponding author: hamzaouirayen2002@yahoo.fr

https://doi.org/10.55251/jmbfs.4889

\section{ARTICLE INFO}

Received 8. 6. 2021

Revised 6. 10. 2021

Accepted 13. 10. 2021

Published 1. 2. 2022

Regular article OPEN $\partial_{\text {ACCESS }}$

\section{ABSTRACT}

The chemical composition of the essential oil of Artemisia herba-alba obtained by hydrodistillation was determined by chromatographic analysis (GC and GC-MS). Seventy-nine components were identified, which accounted for $93.3 \%$ of the total essential oil. The major components were chrysanthenone (24.1\%), camphor (16,2\%), $\alpha$-thujone $(12.8 \%)$ and 1,8-cineole $(9.3 \%)$. The investigated volatile extract showed potency antimicrobial activity, against all the studied bacterial strains (MIC values not exceeding $2 \mu \mathrm{g} / \mathrm{mL}$ ). Also, this oil showed substantial antifungal activity MIC less than $0.5 \mu \mathrm{g} / \mathrm{mL}$ for Fusarium oxysporum and Penicillium expansum. Compared to BHA and BHT which were acted as positive controls, the essential oil was the less active to inhibit the free radical DPPH insofar it failed to reach a scavenging capability of 50\%. The oil was also tested for its corrosion inhibitor of pure aluminum in $\mathrm{HCl}(1 \mathrm{~mol} . / \mathrm{L})$ using electrochemical techniques (potentiodynamic polarization, open circuit potential, and electrochemical impedance spectroscopy) and gravimetric measurements. The results reveal that the inhibition effectiveness was dose-dependent of the oil concentration (maximum corrosion inhibition $=94.3 \%$ for an optimal concentration of $0.8 \mathrm{~g} / \mathrm{L}$ ). These findings suggest that this essential oil may be a new green inhibitor agent against corrosion in hydrogen chloride solution media and could be used on food systems as an effective inhibitor of foodborne pathogens as well as for pharmaceutical applications.

Keywords: Artemisia herba-alba, Essential Oils, Antimicrobial activity, Antioxidant activity, Corrosion, Inhibitor, Metallic Materials hydrochloric acid

\section{INTRODUCTION}

Aluminum exhibits excellent corrosion resistance owing to the formation of a thin protective film of oxide, naturally formed on its surface (Halambek et al., 2013) It is extensively used in industry, but, the major impediments for its use on wider scale are its sensitiveness to be rusted in humid air and dissolved in acidic media (Chauhan et al., 2007). Acid solutions such as hydrogen chloride and sulfuric acids are extensively used in pickling processes of metals which are companioned by significant dissolution of the metals (Hussin et al., 2016; Khadraoui et al..2013). The harmful effect of these acids leads to the use of inhibitors which are judged to be the best method of protection of metals against corrosion. (Chauhan et al., 2007; Khadraoui et al.,2013). Use of natural products such as volatile extracts from plants as corrosion inhibitors have been broadly reported by several authors (Valnet,1984),out with the aim of developing inhibitors that are acceptable to the environment and less expensive. For this purpose, natural products can be considered as the inexhaustible source (Ating et al., 2010). Lately, some studies have experienced the efficacy of plant materials as corrosion inhibitors for aluminum in diverse media (Halambek et al., 2013). The inhibition efficacy of plant extracts is ascribed to the presence of complex organic compounds with diversity of adsorption centers (double bonds, heteroatoms, aromatic rings etc.) (Halambek et al., 2013; Khadraoui et al., 2013; Ating et al., 2010; Hachelef $e t$ al., 2016).

A. herba-alba species are commonly used as a flavoring agent for coffee and in folk medicine due to its multiple therapeutic benefits (Bailey $\boldsymbol{e t}$ al., 1981). It is principally used against the treatment of gastric disturbances and as an antidiabetic agent (Jouad et al., 2001; Mohamedet al.,2010). The chemical composition and biological activity of the plant have been reviewed recently, and the essential oil composition has received ample attention (Mahmoud $\boldsymbol{e t}$ al., 1988; Salido et al., 2004; Dahmani-Hamzaoui et al., (2010, 2015, 2018); Segal et al., 1987). Thus, several researchers have studied several times the volatile components of $A$. herbaalba collected in Spain (Salido et al., 2004) and Algeria (Dahmani-Hamzaoui.,et al 2010, 2015, 2018). Sesquiterpene lactones, flavonoids, some phenolic compounds, and waxes have also been found in the plant(Segal et al., 1987; Dahmani-Hamzaoui et al., 2012). The antibacterial, antifungical and antioxidant activity of A. herba-alba has been evaluated several times (Mahmoud $\boldsymbol{e t}$ al. 1988), the compounds responsible for such activity have been previously isolated (Dahmani-Hamzaoui et al., 2012). Nowadays, to the best of our knowledge, there is no work relating on inhibiting properties of A. herba-alba volatiles on acidic corrosion of aluminum. On the other hand, some studies were published on the use of this plant as anticorrosive agent. (Hechiche et al., 2019;Boudalia et al., 2019; Ouachikh, et al., 2009 ; Benabdellah, et al., 2006 ; Bouyanzer et al., 2004)

The aim of this work is to study for the first time the inhibitory effect on corrosion of aluminum in acidic media by A. herba-alba essential oil as well as its antioxidant and antimicrobial activities. Thus, the diversity of uses of this oil will offer the possibility of choosing one or more preferred compounds for green corrosion inhibitors, the pharmaceutical and food industry. 


\section{MATERIAL AND METHODS}

\section{Vegetable material}

Artemisia herba-alba aerial parts were collected at the blooming stage of the plant in June 2018 from from Boussâada (245Km south-east of Algiers; 35 $13^{\prime} 09^{\prime \prime} \mathrm{N}$, $4^{\circ} 10^{\prime} 54^{\prime \prime}$ E). A Voucher specimen of the plant was filed in the laboratory of plant's Biology in the Faculty of Biological sciences, University of Sciences and Technology Houari Boumediene, Algiers.

\section{Essential oil extraction}

Plant samples were shade dried ( 3 - 6 days) and hydrodistilled, for $3 \mathrm{~h}$. The resulting oil was dried over anhydrous sodium sulphate $\left(\mathrm{Na}_{2} \mathrm{SO}_{4}\right)$, and stored at $4^{\circ} \mathrm{C}$ until use.

\section{Chemicals}

Butylatedhydroxyanisole (BHA), butylated hydroxytoluene (BHT) and 2,2 diphenyl-1-picrylhydrazyl (DPPH), were purchased from Sigma-Aldrich Chemie (Steinheim, Germany), n-alkane standard solution $\left(\mathrm{C}_{7}-\mathrm{C}_{28}\right)$ was obtained from Fluka Chemika (Buchs, Switzerland). Methanol (analytical grade), $\mathrm{Na}_{2} \mathrm{SO}_{4}$, Tween 80, Sabouraud Dextrose Agar (SDA) and Mueller-Hinton (MH) were obtained from Merck (Darmstadt, Germany). The authentic standards for chromatographic analysis were purchased from Sigma-Aldrich (St. Louis, USA), Fluka (Buchs, Switzerland) and Merck (Darmstadt, Germany). The aluminum samples of dimensions $(1.5 \times 1.5 \times 0.5 \mathrm{~cm})$ were cut from an aluminum foil with the following chemical composition (in wt. \%): $0.15 \mathrm{Fe} ; 0.06 \mathrm{Si} ; 0.002 \mathrm{Zn} ; 0.004 \mathrm{Cu}$ and the balance $\mathrm{Al}$. The aggressive solution $(1 \mathrm{~mol} / \mathrm{L} \mathrm{HCl})$ was made from pure-grade $\mathrm{HCl}$ provided by Prolabo Chemical Co and deionized water

\section{Chemical analysis}

For gas chromatography (GC) analysis a Hewlett-Packard 6890N gas chromatograph equipped with a flame ionization detector (FID) was used. The analysis was made by the mean of two columns under the following operating conditions: HP5MS column $(30 \mathrm{~m} \times 0.25 \mathrm{~mm}$ LD., film thickness $0.25 \mu \mathrm{m}$ constant gas flow $0.3 \mathrm{~mL} / \mathrm{min}$ ) vector gas, $\mathrm{N}_{2}$; injector and detector temperatures $250{ }^{\circ} \mathrm{C}$ and $320^{\circ} \mathrm{C}$, respectively; injected volume $0.2 \mu \mathrm{L}$ pure oil; split-less mode; and HP wax column ( $60 \mathrm{~m} \times 0.32 \mathrm{~mm}$ LD., film thickness $0.25 \mu \mathrm{m}$; constant flow $0.9 \mathrm{~mL} / \mathrm{min}$ ); the oven temperature program was $60^{\circ} \mathrm{C}$ for $8 \mathrm{~min}$, rising to $250^{\circ} \mathrm{C}$ at $2{ }^{\circ} \mathrm{C} / \mathrm{min}$, then held for $30 \mathrm{~min}$ at $250{ }^{\circ} \mathrm{C}$. Retention indices were calculated relative to the $\mathrm{C}_{7}-\mathrm{C}_{28} \quad$ n-alkanes injected under the same conditions as the oil. Relative amounts of components are based on FID peak areas without using response factor correction.

Gas chromatography coupled to mass spectrometry (GC/MS) analysis was performed using an Agilent $6890 \mathrm{~N}$ chromatograph. The fused-silica capillary column HP5MS and HP wax (The same as those used in the analysis by GC-FID) were coupled to an Agilent 5973A mass spectrometer. Carrier gas He, injector and detector temperatures, $250{ }^{\circ} \mathrm{C}$ flow rate $0.5 \mathrm{ml} / \mathrm{min}$; split $1: 20$; injection volume $0.1 \mu \mathrm{L}$; oven temperature program for each column is described above for GC; an ionization mode with electronic impact at $70 \mathrm{eV}$ was used over the mass range 35 400 units.

\section{Components identification}

Compound identification was made by comparison of mass spectral fragmentation patterns with those stored in the MS database (NIST 2007 and Wiley 7N), and with mass spectra literature data, and verified by comparison of linear retention indices of the identified compounds with published index data on apolar and polar columns (Adams, 2001; Masada, 1979). Relative percentage quantities of the constituents of the oils were determined from FID chromatograms using the apolar column (HP-5MS).

The results were expressed as the means \pm standard deviation $(n=3)$ by use of Microsoft Excel statistical analysis program.

\section{Antimicrobial activity}

The antimicrobial activity was made by the use of the agar dilution method [NCCLS/CLSI, 2004]. Cultures of the following microorganisms were employed: four Gram-positive bacteria (Bacillus coagulans, Bacillus subtilis, Microccus luteus and Staphylococcus aureus), three Gram-negative bacteria (Agrobacterium tumefaciens, Escherichia coli and Pseudomonas aeruginosa) and five fungi (Mucor ramannianus, Aspergillus ochraceus, Fusarium oxysporum f. sp. albedinis, Penicillium expansum and Fusarium oxysporum f. sp. Lini) and two yeasts (Candida albicans and Saccharomyces cerevisiae). A final concentration of $1 \%(\mathrm{v} / \mathrm{v})$ Tween-80 was added to the agar after autoclaving in order to enhance oil solubility. Briefly, a series of two fold dilutions of the oil ( 0.5 to $75 \mu \mathrm{g} / \mathrm{mL})$ was prepared with Tween-80 (1\% (v/v)). Each Petri dish of $5 \mathrm{~mm}$ in diameter, contains $3 \mathrm{~mL}$ of nutrient agar medium and a known concentration of essential oil was dried prior to inoculation with $2 \mu \mathrm{L}$ of spots containing approximately 3 x 106 cells (for bacteria and yeasts) or spores (for fungi) of each micro-organism. The experiment was repeated twice to confirm the inhibitory effect. Minimum inhibitory concentrations (MIC) were established after $24 \mathrm{~h}$ incubations at $37^{\circ} \mathrm{C}$ for bacteria and yeast and $48 \mathrm{~h}$ for filamentous fungi. The MIC value was determined to be the lowest concentration of oil that could inhibit the visible growth of each micro-organism on the agar plate.

Scavenging of $\mathrm{DPPH}^{\bullet}$ radical activity

The antioxidant activity of the essential oil was determined based on the ability of the tested samples to scavenge the free radical DPPH ${ }^{\bullet}$ (Brand-Williams et al., 1995) by off-line spectrophotometric measurements. Solutions (2.4 mL) of $\mathrm{DPPH}^{\bullet}$ in methanol $\left(10^{-5} \mathrm{M}\right)$ with an absorbance of $0.800 \pm 0.030 \mathrm{AU}$ at $517 \mathrm{~nm}$ were blended with methanolic solutions $(1.2 \mathrm{~mL})$ of samples at differen concentrations (300-1000 $\mu \mathrm{g} / \mathrm{mL})$. Triplicate samples were shaken and allowed to stand for $15 \mathrm{~min}$ in the dark at room temperature, and the decrease of absorbance at 517nm was measured using a Perkin-Elmer Instruments, Nor-walk, CT, USA). The antioxidant activity of the tested samples, expressed as DPPH scavenging percentage was calculated by the following formula:

\section{Radical scavenging $\left.\%=\left[\left(\mathbf{A}_{\mathbf{B}}-\mathbf{A}_{\mathbf{S}}\right) / \mathbf{A}_{\mathbf{B}}\right)\right] \mathbf{x} 100$}

where $A_{B}$ is the absorbance of the blank sample $(t=0)$, and $A_{S}$ is the observance of the tested sample after $15 \mathrm{~min}$.

Sample concentration providing $50 \%$ inhibition $\left(\mathrm{IC}_{50}\right)$ was calculated from the graph plotting inhibition percentage against oil concentration. BHT and BHA were used as positive controls.

\section{Electrochemical measurements}

Electrochemical tests were conducted on aluminum (99.90\%). The inhibitor solution was prepared by disbanding $30 \%(\mathrm{~V} / \mathrm{V})$ of the essential oil in ethanol $(96 \%)$. The concentrations of the essential oil, ranged from 0.2 to $0.8 \mathrm{~g} / \mathrm{L}$, were obtained by dissolving a desired amount of the inhibitor in $\mathrm{HCl}(1 \mathrm{~mol} / \mathrm{L})$ solution. The electrochemical experiments were performed in a conventional three-electrode electrochemical cell, with $\mathrm{Ag} / \mathrm{AgCl} / \mathrm{KCl}$ saturated electrode as reference, platinum plate of $1 \mathrm{~cm}^{2}$ surface size as counter electrode and aluminum sample as a working electrode. Tests were made using an Autolab PGSTAT 30 with FRA2 module (Eco Chemie, Netherlands). Autolab Software version 4.9 monitors the experimental sequences. Thereafter, the GPES 4.9 software (General Purpose Electrochemica System) was used for open circuit potential (OCP) and polarization curve measurements and FRA 4.9 software (Frequency Response Analysis) for impedance data measurements.

\section{Open circuit potential (OCP)}

Open circuit potential were continuously controlled with respect to the saturated $\mathrm{Ag}-\mathrm{AgCl}$ electrode, for $1 \mathrm{~h}$ of immersion in aerated solution of $\mathrm{HCl} 1 \mathrm{M}$, at $303^{\circ} \mathrm{K}$ in absence and attendance of A. herba alba oil with stirring at $100 \mathrm{rpm}$.

\section{Potentiodynamic polarization}

The Tafel plots based on potentiodynamic polarization were recorded by performing a potential sweep of $\pm 300 \mathrm{mV}$ around OCPat a scan rate of $1 \mathrm{mV} . \mathrm{s}^{-1}$ from negative to positive potential values. The extrapolation of the cathodic and anodic Tafel lines, intersect at $\mathrm{E}_{\mathrm{corr}}$ and allows the determination of the corrosion current density, $\left(\mathrm{I}_{\text {corr }}\right)$. The inhibition efficiency was calculated by the polarization method using the following equation:

$$
\eta^{\prime} \%=\left(\frac{I_{\text {corr }}-I_{\text {corr }}^{\prime}}{I_{\text {corr }}}\right) \times 100
$$

Where $I_{\text {corr }}$ and $I_{\text {corr }}^{\prime}$ correspond to the densities of the corrosion current in the absence and presence of Artemisia herba-alba essential oil.

\section{The electrochemical impedance spectroscopy (EIS)}

Measurements of electrochemical impedance spectroscopy (EIS) were carried out at OCP after $1 \mathrm{~h}$ of immersion in the test solution, with sinusoidal voltage amplitude of $10 \mathrm{mV}$ and scan frequency $(f)$ ranging from $100 \mathrm{kHz}$ to $0.01 \mathrm{~Hz}$. The impedance spectra were fitted with the equivalent electrical circuit using the Equivcrt program in FRA software. The inhibition effectiveness was calculated using the equation given by:

$$
\eta \%=\left(\frac{R_{c t}-R_{c t}^{\prime}}{R_{c t}}\right) \times 100
$$

Where $\mathrm{R}_{\mathrm{ct}}$ and $\mathrm{R}_{\mathrm{ct}}$ represent the charges transfer resistances with and without inhibitor, respectively. 


\section{Gravimetric measurements}

Gravimetric measurements were obtained with rectangular aluminum samples, finely mechanically polished using silicon carbide ( $\mathrm{Si}-\mathrm{C}$ ) paper up to 1200 grade under water jet, then ultrasonically degreased in absolute ethanol and ultimately air-dried with acetone. The polished samples were weighted before and after $3 \mathrm{~h}$ of immersion in $100 \mathrm{~mL} \mathrm{HCl}(1 \mathrm{~mol} / \mathrm{L})$ without and along the addition of inhibitor at different concentrations: The tests were carried out three times with good reproducibility. The weight loss of aluminum sample was used to evaluate the corrosion proportion in milligrams per square centimeter per hour.

$$
v=\left(\frac{\Delta m}{s \times t}\right)
$$

Where $\Delta m$ is the weight loss, $\mathrm{t}$ is the immersion duration and s the sample surface

$$
\eta^{\prime \prime} \%=\left(\frac{v_{\text {corr }}-v_{\text {corr }}^{\prime}}{v_{\text {corr }}}\right) \times 100
$$

Where $v_{\text {corr }}$ and $v_{\text {corr }}^{\prime}$ are the corrosion rates without and with solution, respectively.

\section{RESULTS AND DISCUSSION}

\section{Chemical composition}

The yields of essential oil extracted from A. herba-alba, calculated from air-dried vegetal material (w/w) are to $0.93 \pm 0.06 \%$. Chromatographic analysis by GC and GC-MS of A. herba-alba essential oil isolated by hydrodistillation led to the identification of 79 compounds (Fig1, Table I), which represent $93.3 \%$ of the total oil.

The dominant constituents of the oil were chrysanthenone $(24.1 \%)$, camphor (16.2\%), $\alpha$-thujone $(12.8 \%), 1,8$-cineole $(9.3 \%)$ and $\beta$-thujone $(4.8 \%)$ (Fig 2) Camphene, sabinene and $\alpha$-pinene have so far only been reported as traces. In this work they accounted $(2.4 \%, 1.5 \%$ and $1.0 \%)$, respectively.

Camphor is the most prominent component in the essential oil of Boussaada (49.3\%) and Djelfa (32\%) of Algeria (Dahmani-Hamzaoui et al., 2010; Lakehal et al., 2017). Chrysanthenone is very important in the essential oil of Bordj Bou Arrérridj (43.8\%), M'sila (34.3\%) (Dahmani-Hamzaoui et al., 2010), southern Spain (36.40\%) (Salido et al., 2004) and Tunisia (17.37\%) (Mighri et al., 2010). This compound could come from the hydrolysis and then the oxidation of cischrysanthenyl acetate (Giordani et al.,2008)

1,8-Cineole (5.61\%) is the main compound in southern Spain essential oil (41.0\%) (Salido et al., 2004), Ichemoul in Algeria (Bezza et al., 2010) and southern Tunisia (20.00\%) (Akrout, 2004)

\begin{tabular}{|c|c|c|c|c|c|}
\hline $\mathbf{N}^{\circ}$ & Compound & $\mathbf{R I}_{\mathbf{1}}$ & $\mathbf{R I}_{2}$ & $\%$ & Identification \\
\hline 1 & Santalinatriene $\mathrm{a}^{\mathrm{a}, \mathrm{i}, \mathrm{j}, \mathrm{l}}$ & 908 & 1028 & $0.7 \pm 0.03$ & MS,RI, Std \\
\hline 2 & Tricyclene ${ }^{\mathrm{a}, \mathrm{g}, \mathrm{h}, \mathrm{i}, \mathrm{j}}$ & 921 & 1007 & $0.2 \pm 0.01$ & MS,RI \\
\hline 3 & $\alpha$-Thujene ${ }^{\mathrm{a}, \mathrm{d}, \mathrm{e}, \mathrm{g}}$ & 926 & 1027 & $0.1 \pm 0.01$ & MS,RI \\
\hline 4 & $\alpha$-Pinene ${ }^{\mathrm{a}, \mathrm{b}, \mathrm{c}, \mathrm{f}, \mathrm{g}, \mathrm{h}, \mathrm{i}, \mathrm{j}, \mathrm{k}, \mathrm{k}}$ & 933 & 1022 & $1.0 \pm 0.05$ & MS,RI, Std \\
\hline 5 & Camphene $e^{\mathrm{c}, \mathrm{d}, \mathrm{e}, \mathrm{f}, \mathrm{g}, \mathrm{h}, \mathrm{i}, \mathrm{j}}$ & 947 & 1061 & $2.4 \pm 0.11$ & MS,RI, Std \\
\hline 6 & Thuja-2,4(10)-diene $\mathrm{g.j}$ & 948 & 1120 & $\operatorname{tr}$ & MS,RI \\
\hline 7 & 1-Octen-3-ol ${ }^{\mathrm{h}, \mathrm{i}}$ & 971 & 1431 & $\operatorname{tr}$ & MS,RI, Std \\
\hline 8 & Sabinene $e^{\mathrm{a}, \mathrm{b}, \mathrm{d}, \mathrm{h}, \mathrm{i}, \mathrm{j}, \mathrm{k}}$ & 971 & 1116 & $1.5 \pm 0.08$ & MS,RI, Std \\
\hline 9 & $\beta$-Pinene $e^{a, b, c, d, e, f}$ & 973 & 1100 & $0.3 \pm 0.01$ & MS,RI, Std \\
\hline 10 & 1,3,5-Trimethylbenzene $\mathrm{a}^{\mathrm{a}, \mathrm{h}}$ & 986 & 1257 & $\operatorname{tr}$ & MS,RI \\
\hline 11 & Myrcene ${ }^{\mathrm{a}, \mathrm{b}, \mathrm{g}, \mathrm{h}, \mathrm{k}}$ & 992 & 1160 & $1.5 \pm 0.08$ & MS,RI, Std \\
\hline 12 & $\alpha$-Phellandrene ${ }^{\mathrm{d}, \mathrm{f}, \mathrm{g}, \mathrm{h}}$ & 1001 & 1163 & $0.1 \pm 0.01$ & MS,RI, Std \\
\hline 13 & $\alpha$-Terpinene ${ }^{\mathrm{a}, \mathrm{c}, \mathrm{d}, \mathrm{h}, \mathrm{i}, \mathrm{j}, \mathrm{k}}$ & 1015 & 1179 & $0.4 \pm 0.01$ & MS,RI, Std \\
\hline 14 & 1,2,3-Trimethylbenzene $\mathrm{a}^{\mathrm{a}, \mathrm{h}}$ & 1015 & 1287 & $\operatorname{tr}$ & MS,RI \\
\hline 15 & $\beta$-Phellandrene ${ }^{\mathrm{a}, \mathrm{g}}$ & 1023 & 1207 & $\operatorname{tr}$ & MS,RI, Std \\
\hline 16 & p-Cymene $e^{\mathrm{d}, \mathrm{e}, \mathrm{f}, \mathrm{g}, \mathrm{h}, \mathrm{i}, \mathrm{j}, \mathrm{k}}$ & 1024 & 1256 & $0.3 \pm 0.01$ & MS,RI, Std \\
\hline 17 & 1,8 -Cineole ${ }^{\mathrm{a}, \mathrm{b}, \mathrm{c}, \mathrm{d}, \mathrm{e}, \mathrm{f}, \mathrm{g}, \mathrm{h}, \mathrm{i}, \mathrm{j}, \mathrm{k}}$ & 1031 & 1204 & $5.6 \pm 0.13$ & MS,RI, Std \\
\hline 18 & Santalina alcohol ${ }^{\mathrm{a}, \mathrm{e}, \mathrm{f}, \mathrm{g}, \mathrm{i}}$ & 1037 & - & $0.5 \pm 0.02$ & MS,RI \\
\hline 19 & $\beta$-Oimene $\mathrm{e}^{\mathrm{d}, \mathrm{f}, \mathrm{h}, \mathrm{g}}$ & 1041 & 1214 & $\operatorname{tr}$ & MS,RI, Std \\
\hline 20 & $\gamma$-Terpinene $\mathrm{e}^{\mathrm{a}, \mathrm{c}, \mathrm{d}, \mathrm{e}, \mathrm{f}, \mathrm{g}, \mathrm{h}, \mathrm{i}, \mathrm{j}, \mathrm{k}}$ & 1058 & 1238 & $0.5 \pm 0.02$ & MS,RI, Std \\
\hline 21 & cis-Sabinene hydrate $e^{e, i, j}$ & 1068 & 1469 & $0.4 \pm 0.01$ & MS,RI \\
\hline 22 & Artemisia alcohol ${ }^{\mathrm{a}, \mathrm{e}, \mathrm{f}, \mathrm{g}, \mathrm{i}}$ & 1085 & 1481 & $0.8 \pm 0.03$ & MS,RI \\
\hline 23 & Terpinolene $\mathrm{e}^{\mathrm{a}, \mathrm{c}, \mathrm{d}, \mathrm{f}, \mathrm{g}, \mathrm{h}}$ & 1087 & 1277 & $\operatorname{tr}$ & MS,RI \\
\hline 24 & trans-sabinene hydrate ${ }^{\mathrm{a}, \mathrm{c}, \mathrm{g}}$ & 1095 & 1450 & $\operatorname{tr}$ & MS,RI \\
\hline 25 & Filifolone $^{\mathrm{a}, \mathrm{h}}$ & 1103 & 1423 & $0.1 \pm 0.01$ & MS,RI \\
\hline 26 & $\alpha$-Thujone $e^{\mathrm{a}, \mathrm{b}, \mathrm{e}, \mathrm{f}, \mathrm{g}, \mathrm{h}, \mathrm{i}, \mathrm{j}, \mathrm{k}}$ & 1106 & 1409 & $12.8 \pm 0.16$ & MS,RI, Std \\
\hline 27 & $\beta$-Thujone $\mathrm{a}^{\mathrm{a}, \mathrm{b}, \mathrm{e}, \mathrm{f}, \mathrm{g}, \mathrm{h}, \mathrm{i}, \mathrm{j}, \mathrm{k}}$ & 1118 & 1422 & $4.8 \pm 0.10$ & MS,RI \\
\hline 28 & Chrysanthenone $\mathrm{e}^{\mathrm{a}, \mathrm{c}, \mathrm{d}, \mathrm{e}, \mathrm{f}, \mathrm{g}, \mathrm{h}, \mathrm{i}, \mathrm{j}, \mathrm{k}, \mathrm{k}}$ & 1130 & 1505 & $24.1 \pm 0.45$ & MS,RI \\
\hline 29 & trans-Pinocarveol ${ }^{\mathrm{a}, \mathrm{b}, \mathrm{d}, \mathrm{e}, \mathrm{f}, \mathrm{g}, \mathrm{h}}$ & 1134 & 1622 & $0.6 \pm 0.03$ & MS,RI, Std \\
\hline 30 & Camphor $^{\mathrm{a}, \mathrm{b}, \mathrm{c}, \mathrm{d}, \mathrm{e}, \mathrm{f}, \mathrm{g}, \mathrm{h}, \mathrm{i}, \mathrm{j}, \mathrm{k}}$ & 1148 & 1514 & $16.2 \pm 0.24$ & MS,RI, Std \\
\hline 31 & trans-Verbenol & 1149 & 1648 & $0.2 \pm 0.01$ & MS,RI \\
\hline 32 & Isothujanol $^{\mathrm{b}, \mathrm{h}}$ & 1155 & & $0.1 \pm 0.01$ & MS,RI \\
\hline
\end{tabular}

Table 1 Composition (\%) of $A$. herba-alba oil isolated by hydrodistillation
The $\alpha$-thujone $(12.82 \%)$ is the major component in Tunisia (44.0\%) and Jordan (16.0\%) essential oils (Akrout, 2004;Hudaib et al., 2003). On the other hand, it is present in variable compositions in the regions of Algeria (1.50 to 35.1\%) (Khadraoui et al., 2013;Dahmani, 2010). This compound has been reported to have antibacterial, emmenagogue, insecticide and larvicide properties (Zouari $\boldsymbol{e t}$ al., 2010).

The oil was dominated by ketones $(61.6 \%)$, followed by alcohols $(15.0 \%)$. Mono and sesquiterpenic hydrocarbons represented only $8.9 \%$ and $4.8 \%$ of total oil respectively.

The oil herein studied is characterized by the chrysanthenone/camphor/ $\alpha$-thujone chemotype, while different Algerian chemotypes, such as camphor (region of Boussâada and Djelfa), camphor/1,8-cineole (Medjedel region), camphor/ $\alpha$ thujone/1,8-cineole/chrysanthenone (Djelfa region), camphor/ $\alpha$-thujone and camphor/trans-pinocarveol/chrysanthenone/ $\alpha$-thujone and 1,8-cineole (regions of Batna and M'sila), $\alpha$-thujone/ $\beta$-thujone/1,8-cineole, $\alpha$-thujone/chrysanthenone (Draâ Ech Chih), chrysanthenone/camphor/ $\alpha$-thujone (Bordj Bou Arreridj) and davanone (region of Bordj Ghedir and Djelfa) have already been reported (Dahmani-Hamzaoui et al., (2010, 2018); Dahmani, 2010). Previously, Salido et al., (2004) reported that most species of Spanish A. herba-alba oils are characterized by 1,8-cineole and derivatives of bornane skeletons (camphor, borneol, camphene, etc.)

In general, this variation of the chemical composition of A. herba-alba can be attributed to the techniques used for extraction and exogenous factors: the sunshine and the nature and the composition of the soil.

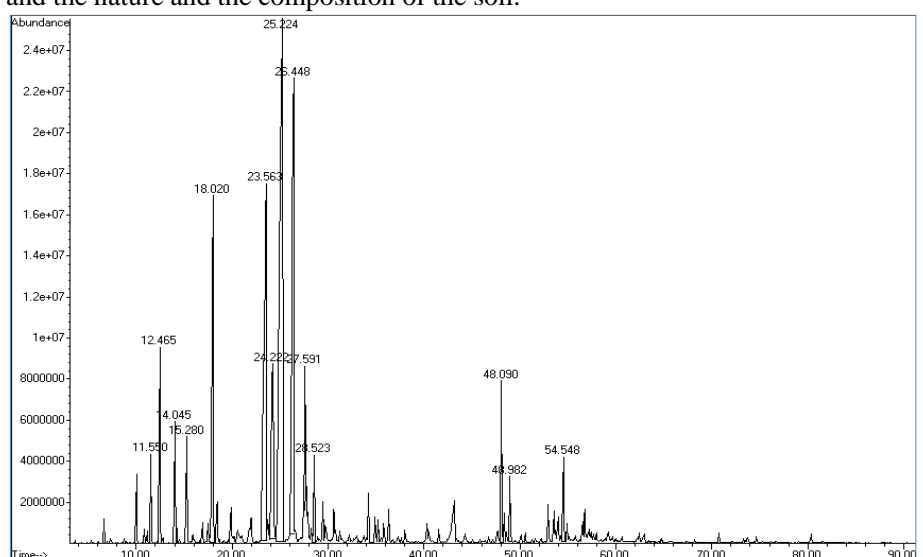

Figure 1 Chromatogram of A. herba Alba essential oil 


\begin{tabular}{|c|c|c|c|c|c|}
\hline 33 & Sabina ketone $e^{\mathrm{g}, \mathrm{i}, \mathrm{j}}$ & 1156 & - & $0.1 \pm 0.01$ & MS,RI \\
\hline 34 & Pinocarvone $\mathrm{e}^{\mathrm{a}, \mathrm{e}, \mathrm{g}, \mathrm{h}, \mathrm{i}, \mathrm{j}, \mathrm{k}}$ & 1165 & 1570 & $0.5 \pm 0.02$ & MS,RI, Std \\
\hline 35 & Borneol $^{\mathrm{a}, \mathrm{b}, \mathrm{c}, \mathrm{d}, \mathrm{e}, \mathrm{f}, \mathrm{g}, \mathrm{h}, \mathrm{i}, \mathrm{k}}$ & 1169 & 1675 & $0.8 \pm 0.02$ & MS,RI, Std \\
\hline 36 & Terpinen 4-ol ${ }^{\mathrm{a}, \mathrm{c}, \mathrm{d}, \mathrm{e}, \mathrm{f}, \mathrm{g}, \mathrm{h}, \mathrm{i}, \mathrm{j}, \mathrm{k}}$ & 1175 & 1589 & $0.6 \pm 0.02$ & MS,RI, Std \\
\hline 37 & Santalinyl acetate ${ }^{\mathrm{a}, \mathrm{e}}$ & 1176 & - & $0.1 \pm 0.01$ & MS,RI \\
\hline 38 & Thuj-3-en-10-al ${ }^{\mathrm{g}}$ & 1185 & 1566 & $0.1 \pm 0.01$ & MS,RI \\
\hline 39 & p-Cymen-8-ol ${ }^{\mathrm{a}, \mathrm{c}, \mathrm{d}, \mathrm{e}, \mathrm{g}, \mathrm{i}}$ & 1187 & 1830 & $0.6 \pm 0.02$ & MS,RI \\
\hline 40 & $\alpha$-Terpineol ${ }^{\mathrm{a}, \mathrm{b}, \mathrm{c}, \mathrm{d}, \mathrm{e}, \mathrm{g}, \mathrm{h}, \mathrm{i}, \mathrm{k}}$ & 1191 & 1672 & $0.3 \pm 0.01$ & MS,RI, Std \\
\hline 41 & Myrtenol $^{\mathrm{a}, \mathrm{c}, \mathrm{e}, \mathrm{f}, \mathrm{g}, \mathrm{h}}$ & 1199 & 1774 & $0.5 \pm 0.02$ & MS,RI, Std \\
\hline 42 & trans-Piperitol ${ }^{\mathrm{a}, \mathrm{b}, \mathrm{c}, \mathrm{d}, \mathrm{e}, \mathrm{f}, \mathrm{g}, \mathrm{h}}$ & 1208 & 1740 & $0.7 \pm 0.03$ & MS,RI \\
\hline 43 & Verbenone $\mathrm{a}^{\mathrm{a}, \mathrm{b}, \mathrm{f}, \mathrm{g}, \mathrm{h}, \mathrm{i}, \mathrm{j}, \mathrm{k}}$ & 1209 & 1683 & $0.1 \pm 0.02$ & MS,RI \\
\hline 44 & trans-Carveol ${ }^{\mathrm{a}, \mathrm{f}, \mathrm{g}, \mathrm{h}}$ & 1218 & 1820 & $0.1 \pm 0.01$ & MS,RI \\
\hline 45 & cis-Carveol $^{\mathrm{a}, \mathrm{g}, \mathrm{h}, \mathrm{j}}$ & 1229 & 1848 & $0.1 \pm 0.01$ & MS,RI \\
\hline 46 & Cumin aldehyde $\mathrm{a}^{\mathrm{a}, \mathrm{c}, \mathrm{d}, \mathrm{g}, \mathrm{h}}$ & 1243 & 1770 & $0.1 \pm 0.01$ & MS,RI, Std \\
\hline 47 & trans-Ocimenone & 1244 & 1813 & $\operatorname{tr}$ & MS,RI \\
\hline 48 & Carvone $\mathrm{e}^{\mathrm{a}, \mathrm{f}, \mathrm{g}, \mathrm{h}, \mathrm{i}, \mathrm{j}}$ & 1244 & 1721 & $0.1 \pm 0.01$ & MS,RI, Std \\
\hline 49 & Carvotanacetone $\mathrm{e}^{\mathrm{a}, \mathrm{g}}$ & 1255 & 1713 & $0.2 \pm 0.01$ & MS,RI \\
\hline 50 & Piperitone $\mathrm{e}^{\mathrm{a}, \mathrm{b}, \mathrm{c}, \mathrm{f}, \mathrm{g}, \mathrm{h}}$ & 1255 & 1710 & $0.1 \pm 0.01$ & MS,RI, Std \\
\hline 51 & cis-Chrysanthenyl acetate $\mathrm{e}^{\mathrm{a}, \mathrm{d}, \mathrm{e}, \mathrm{g}, \mathrm{h}, \mathrm{i}, \mathrm{j}, \mathrm{k}, \mathrm{k}}$ & 1262 & 1605 & $0.9 \pm 0.04$ & MS,RI \\
\hline 52 & Isobornyl acétate & 1286 & 1572 & $0.3 \pm 0.01$ & MS,RI \\
\hline 53 & Bornyl acetate $\mathrm{a}^{\mathrm{a}, \mathrm{b}, \mathrm{c}, \mathrm{d}, \mathrm{e}, \mathrm{g}, \mathrm{h}, \mathrm{i}, \mathrm{j}}$ & 1294 & & $0.5 \pm 0.02$ & MS,RI, Std \\
\hline 54 & Carvacrol $^{\mathrm{d}, \mathrm{f}, \mathrm{i}}$ & 1299 & 2150 & $0.1 \pm 0.01$ & MS,RI, Std \\
\hline 55 & Cis-Pinocarvyl acétate & 1313 & & $0.1 \pm 0.01$ & MS,RI \\
\hline 56 & 1,6-Dimethylhepta-1,3,5-triene & 1321 & & $0.2 \pm 0.01$ & MS,RI \\
\hline 57 & Dihydro Carveol Acetate & 1356 & & $0.4 \pm 0.01$ & MS,RI \\
\hline 58 & Eugenol $^{\mathrm{d}, \mathrm{g}, \mathrm{h}, \mathrm{k}}$ & 1359 & 2155 & $0.2 \pm 0.01$ & MS,RI \\
\hline 59 & $\alpha-$-Copaene $\mathrm{e}^{\mathrm{a}, \mathrm{d}, \mathrm{e}, \mathrm{f}, \mathrm{g}, \mathrm{h}, \mathrm{i}}$ & 1375 & 1476 & $0.2 \pm 0.01$ & MS,RI \\
\hline 60 & cis-Jasmone $\mathrm{e}^{\mathrm{g}, \mathrm{i}}$ & 1398 & 1932 & $1.5 \pm 0.07$ & MS,RI \\
\hline 61 & Methyl eugenol ${ }^{\mathrm{a}, \mathrm{g}, \mathrm{h}}$ & 1399 & 2000 & $0.2 \pm 0.01$ & MS,RI \\
\hline 62 & $\beta$-Caryophyllene $\mathrm{e}^{\mathrm{a}, \mathrm{c}, \mathrm{d}, \mathrm{f}, \mathrm{h}, \mathrm{k}}$ & 1422 & 1594 & $0.2 \pm 0.01$ & MS,RI, Std \\
\hline 63 & Aromadendrene $\mathrm{g}^{\mathrm{g}}$ & 1442 & 1615 & $0.2 \pm 0.01$ & MS,RI \\
\hline 64 & (E)-Ethyl cinnamate ${ }^{\mathrm{f}, \mathrm{g}}$ & 1471 & & $0.2 \pm 0.01$ & MS,RI, Std \\
\hline 65 & $\beta$-Chamigrene ${ }^{\mathrm{g}}$ & 1477 & & $\operatorname{tr}$ & MS,RI \\
\hline 66 & $\gamma$-Murolene $\mathrm{e}^{\mathrm{a}, \mathrm{d}, \mathrm{g}, \mathrm{h}, \mathrm{k}}$ & 1480 & 1691 & $2.3 \pm 0.09$ & MS,RI \\
\hline 67 & Bicyclogermacrene ${ }^{g}$ & 1500 & 1718 & $0.2 \pm 0.01$ & MS,RI \\
\hline 68 & $\alpha$ - Muurolene ${ }^{\mathrm{a}, \mathrm{f}, \mathrm{g}, \mathrm{h}}$ & 1503 & 1706 & $\operatorname{tr}$ & MS,RI, Std \\
\hline 69 & Cubebol $^{\mathrm{g}}$ & 1516 & 1926 & $0.1 \pm 0.01$ & MS,RI \\
\hline 70 & $\delta$-Cadinene $\mathrm{e}^{\mathrm{a}, \mathrm{c}, \mathrm{d}, \mathrm{f}, \mathrm{h}, \mathrm{k}}$ & 1523 & 1742 & $0.1 \pm 0.01$ & MS,RI \\
\hline 71 & Cadina-1,4-diene & 1533 & 2056 & $0.9 \pm 0.06$ & MS,RI \\
\hline 72 & Ledol $^{\mathrm{a}, \mathrm{j}}$ & 1563 & 2020 & $\operatorname{tr}$ & MS,RI \\
\hline 73 & trans-Nerolidol ${ }^{\mathrm{d}, \mathrm{f}, \mathrm{g}, \mathrm{i}}$ & 1564 & 2031 & $\operatorname{tr}$ & MS,RI \\
\hline 74 & Palustrol & 1566 & & $0.5 \pm 0.02$ & MS,RI \\
\hline 75 & Spathulenol ${ }^{\mathrm{b}, \mathrm{d}, \mathrm{f}, \mathrm{g}, \mathrm{h}, \mathrm{i}, \mathrm{k}}$ & 1578 & 2115 & $0.2 \pm 0.01$ & MS,RI, Std \\
\hline 76 & Caryophyllene oxide ${ }^{\mathrm{d}, \mathrm{f}, \mathrm{g}, \mathrm{h}, \mathrm{i}, \mathrm{k}}$ & 1583 & 1975 & $0.4 \pm 0.01$ & MS,RI, Std \\
\hline 77 & $\beta$-Copaen $4-\alpha$ ol $^{\mathrm{g}}$ & 1588 & 2140 & $0.6 \pm 0.01$ & MS,RI \\
\hline 78 & Viridiflorol ${ }^{\mathrm{f}, \mathrm{g}}$ & 1593 & 2075 & $1.2 \pm 0.08$ & MS,RI \\
\hline 79 & Cedrol & 1600 & & $0.2 \pm 0.01$ & MS,RI \\
\hline \multicolumn{2}{|c|}{ Monoterpene hydrocarbons } & \multicolumn{3}{|c|}{8.9} & \\
\hline \multicolumn{2}{|c|}{ Sesquiterpene hydrocarbons } & \multicolumn{3}{|c|}{$\begin{array}{l}0.9 \\
4.7\end{array}$} & \\
\hline \multicolumn{2}{|c|}{ Alcohols } & \multicolumn{3}{|c|}{15.0} & \\
\hline \multicolumn{2}{|c|}{ Ketones } & \multicolumn{3}{|c|}{61.6} & \\
\hline \multicolumn{2}{|c|}{ Ether oxides } & \multicolumn{2}{|c|}{0.5} & & \\
\hline \multicolumn{2}{|c|}{ Esters } & \multicolumn{3}{|c|}{2.4} & \\
\hline \multicolumn{2}{|c|}{ Others } & \multicolumn{3}{|c|}{0.2} & \\
\hline \multicolumn{2}{|c|}{ Identified components $(\%)$} & \multicolumn{3}{|c|}{93.3} & \\
\hline
\end{tabular}

Components listed in order of their elution from the HP 5MS column; $\mathrm{tr}=$ trace (amount< $0.05 \%$ ); $\mathrm{RI}_{1}$ and $\mathrm{RI}_{2}$ : Linear retention indices relative to HP5-MS and HP-Wax capillary columns, respectively; Values are the averages of three measurements \pm SD; RI, comparison of retentions indices with those of published data; MS, comparison of the mass spectra of peaks with MS libraries and published data; Std, comparison of RI and mass spectra with that of with authentic compound (standard).

a : (Benjilali., 1980, 1981); b : (Boutekdjiret, 1992); c : (Feuerstein, 1988); d : (Salido., 2001, 2004); e : (Feuerstein., 1986; Segal., 1987); f : (Dob, 2006),

g : (Dahmani-Hamzaoui.,2010, 2015, 2018); h: (Vernin., 1994, 1995, 2001); i: (Haouari, 2009); j: (Paolini, 2010); k: (Bezza, 2010).

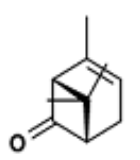

chrysanthenone

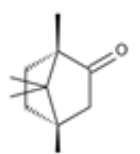

camphor

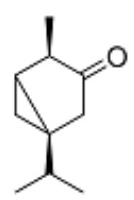

a-thujon

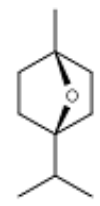

1,8-cineole

Figure 2 Major compounds of A. herba-alba essential oil (Bousâada region)

\section{Antimicrobial activity}

The MICs of essential oil from A. herba-alba against fourteen species of microorganisms by the agar dilution method are summarized in Table $\mathbf{2}$. It shows a good inhibitory potency against all tested microorganisms (MIC not exceeding 2 $\mu \mathrm{g} / \mathrm{mL}$ ) except for Aspergillus ochraceus $(\mathrm{MIC}=5 \mu \mathrm{g} / \mathrm{mL})$. The data obtained from the agar dilution method using A. herba-alba essential oil indicated that Bacillus coagulans, Staphylococcus aureus, Agrobacterium tumefaciens, Fusarium

oxysporum, Penicillium expansum and Pseudomonas aeruginosa were the most sensitive microorganisms tested (MIC $<0.5 \mu \mathrm{g} / \mathrm{mL}$ ).

Globally, the studied oil exhibited a wide antimicrobial spectrum and exerted strong microbial growth-inhibiting properties against all microorganisms The essential oil antimicrobial activity could be ascribed to its major constituents as chrysanthenone, $\alpha$-thujone and camphor (Mahmoud et al., 1988). However, according to some authors, components present at lower concentrations might be engaged in some type of synergy with the other compounds (Oussalah et al., 2007; Vagionas et al., 2007). The biological activity of any essential oil is intimately linked to its chemical composition, the functional groups of the major components and the possible synergistic effects of these constituents, it is likely that minority compounds act in synergy; in this way, the value of the essential oil is due to all of these components (Andreani et al., 2016; Salhi et al., 2017). 
Table 2 MICs $(\boldsymbol{\mu g} / \mathbf{m L})$ of Artemisia herba-alba essential oil

\begin{tabular}{lc}
\hline Microorganism & $\begin{array}{c}\text { Minimal inhibitory concentration } \\
(\boldsymbol{\mu g} / \mathbf{m} \mathbf{L})\end{array}$ \\
\hline Bacteria & 1 \\
\hline Bacillus subtilis ATCC6663 & $<0.5$ \\
Bacillus coagulans CIP6625 & 2 \\
Microccus luteus ATCC9314 & $<0.5$ \\
Staphylococcus aureus CIP7625 & $<0.5$ \\
Agrobacterium tumefaciens $\mathrm{N}^{\circ} 2410$ & 0.5 \\
Escherichia coli CIP54.8 & $<0.5$ \\
Pseudomonas aeruginosa CIPA22 & \\
\hline Fungi & 2 \\
Mucor ramannianus NRRL1829 & 5 \\
Aspergillus ochraceus & $<0.5$ \\
Fusariumox ysporum f.sp. albedinis & $<0.5$ \\
CURZA & 1 \\
Penicillium expansum 8932 & Fusarium oxysporum f. sp. lini \\
CINRA &
\end{tabular}

CINRA

Yeast

Candida albicans CLM $2-5$

Saccharomyces cerevisiae

ATCC4226

ATCC: American Type Culture Collection; CURZA: collection de l'Unité de Recherche sur les Zones Arides (Alger) ; CIP: collection de l'Institut Pasteur de Paris, France; CINRA: collection of the National Institute of Agronomic Research of Dijon, France;

NRRL: Northern Regional Center, Peoria (U.S.A) ; CLM: collection of the Microbiology Laboratory of ENS Kouba, Algiers

\section{Scavenging of radical $\mathrm{DPPH}^{\bullet}$}

The action of antioxidants on the scavenging of DPPH radicals comes from their ability to donate hydrogen. DPPH is a stable free radical which can accept hydrogen radical or an electron to become a stable diamagnetic molecule. During the interaction of an antioxidant with DPPH, there will be an electron or atom transfer to DPPH what leads to the neutralization of its free radical character and convert it to 1-1 diphenyl-2-picryl hydrazine which will result in a decrease of the degree of coloration which indicates the activity of scavenging the antioxidant. In this study the essential oil had significant scavenging effect on the DPPH radical which was dose dependent of the concentration (Table 3).

Table $3 \mathrm{DPPH}^{\bullet}$ radical scavenging activity $(\%)$ and $\mathrm{IC}_{50}(\mu \mathrm{g} / \mathrm{mL})$ of $A$. herba alba essential oil

\begin{tabular}{|c|c|c|c|c|c|c|}
\hline \multirow{3}{*}{ Sample } & \multicolumn{5}{|c|}{ Concentration $(\mu \mathrm{g} / \mathrm{mL})$} & \multirow[b]{2}{*}{ IC $_{50}$} \\
\hline & 300 & 400 & 500 & 700 & 1000 & \\
\hline & \multicolumn{5}{|c|}{ Radical scavenging ${ }^{\mathrm{a}} \pm \mathbf{0 . 0 5}$} & \\
\hline $\mathrm{HE}$ & 37.20 & 38.84 & 41.46 & 44.15 & 46.34 & NA \\
\hline BHA & & & & & & 41.38 \\
\hline BHT & & & & & & 24.91 \\
\hline
\end{tabular}

${ }^{a}$ Values are means of three independent replicates with RSD is less than 1\%; NA : Value not available in the gamut of the experimented concentrations.

Thus, there was an increase of the percent of radical scavenging effect of essential oil on DPPH with the increase of the concentrations of the oil from 300-1000 $\mu \mathrm{g} / \mathrm{mL}$. The inhibition rate of the DPPH radical ranged from $37.20 \%$ (for $300 \mu \mathrm{g} /$ $\mathrm{mL}$ ) to $46.34 \%$ (for $1000 \mu \mathrm{g} / \mathrm{mL}$ ).

However, the oil could not reach the $\mathrm{IC}_{50}$ in the range of concentrations studied (300-1000 $\mu \mathrm{g} / \mathrm{L})$. For all concentrations, the essential oil showed lower percentage of inhibition of DPPH than the standards BHT and BHA). The relative low DPPH radical scavenging activity of this oil compared to those of BHT and BHA could be attributed to the absence of phenolic components such as thymol, carvacrol, eugenol, which play an important role in the antioxidant activity of an oil (Hazzit et al., 2009; Hadjadj et al., 2020).

\section{Electrochemical measurements}

\section{Open circuit potential (OCP)}

Fig 3 displays the temporal evolution of OCP with and without the presence of the essential oil. There is no obvious influence of the essential oil on the open-circuit potential even if a very slight evolution can be observed to more negative values. However, on these chronopotentiograms, fluctuations in potential are observed which originate from an activating action of the material, probably because of the presence of $\mathrm{Cl}^{-}$

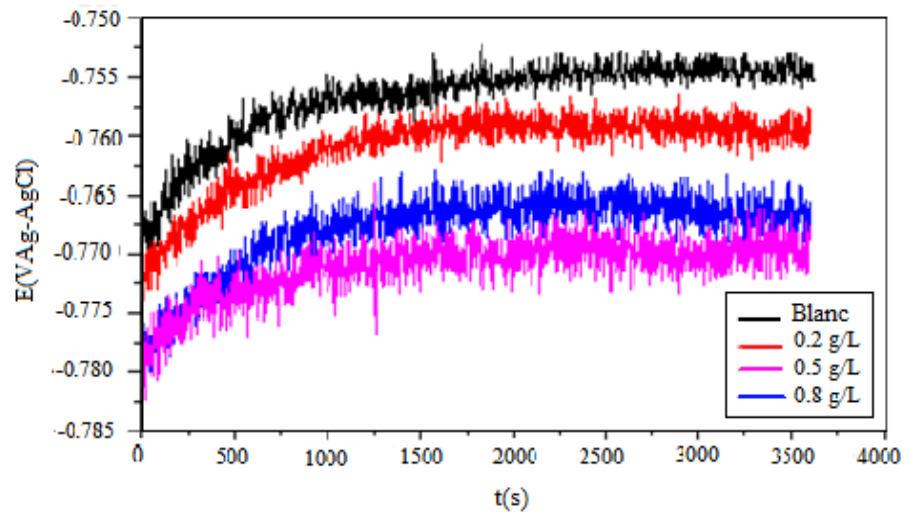

Figure 3OCP-time curves of $\mathrm{Al}$ in $\mathrm{HCl}(1 \mathrm{~mol} . / \mathrm{L})$ solution with and withou essential oil

\section{Potentiodynamic curves}

The curves of Potentiodynamic polarization for aluminum in $\mathrm{HCl}$ solution (1 $\mathrm{mol} . / \mathrm{L}$ ) at $303^{\circ} \mathrm{K}$, without and with various concentrations of $A$. herba-alba oil are shown in Fig 4. As can be seen, in the absence of oil from A. herba-alba, there was no passive film formation during the anodic polarization because there is no plateau on the anode curves. Indeed, according to the Pourbaix diagram, aluminum is passive for $\mathrm{pH}$ ranging from 4 to 8.5 . Beyond these limits, aluminum undergoes corrosion in aqueous solutions because its oxides are soluble, especially in hydrochloric medium where the formation of $\mathrm{Al}^{3+}$ ions in the former and $\mathrm{AlO}_{2}^{-}$ ions in the latter occurs (Branzoi et al., 2003). There is no difference in the anodic portion of the polarization curve with the addition of $A$. herba-alba oil. The shape of the potential-intensity curves seems to indicate that the Tafel relationship is followed, showing that both anodic and cathodic reactions are activationcontrolled.

If we consider the cathode branches of the tafel plots, the aluminum corrosion potential moved 2-18 $\mathrm{mV}$ cathodically in comparison to the control (blank) and also a modification in cathodic Tafel slopes were noted. It is well admitted that an inhibitor is classified as anodic or cathodic type if the shift in potential corrosion is upper than $85 \mathrm{mV}$ with respect to potential of corrosion of the control. (Yanet al., 2008). This indicates that $A$. herba-alba oil acts like mixed-type inhibitor with predominating cathodic efficiency. The cathodic Tafel plot (Fig 4) provided parallel lines denoting that the addition of A.herba-alba oil to the $\operatorname{HCl}\left(1 \mathrm{~mol} . \mathrm{L}^{-1}\right)$ solution did not change the mechanism of hydrogen evolution and the reduction of $\mathrm{H}+$ ions at the aluminum surface occur principally through a charge transfer mechanism. The extract molecules were firstly adsorbed on the metal surface and blocked the reaction sites of the aluminum surface. Thus, the surface area disposable for $\mathrm{H}^{+}$ions has been reduced, justifying the reduction of the cathodic current while the reaction mechanism itself remains unchanged (Solmaz et al., 2008). A larger coverage of the surface by the extract was got in solutions with the higher concentrations.

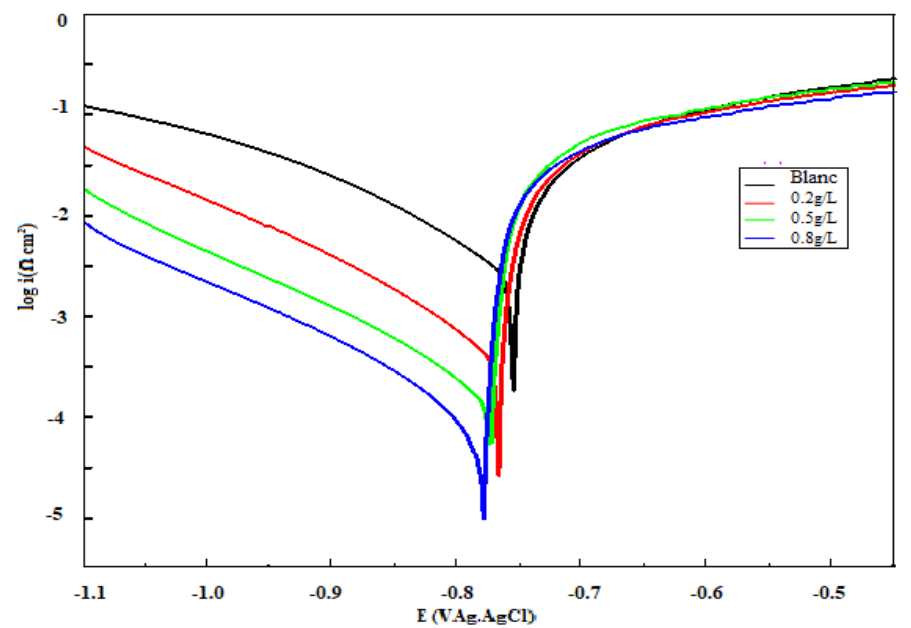

Figure 4 Polarization curves for aluminum in $\mathrm{HCl}(1 \mathrm{~mol} . / \mathrm{L})$ solution with and without $A$.herba-alba oil, $v=1 \mathrm{mV} / \mathrm{s}, T=303^{\circ} \mathrm{K}$

Cathodic Tafel slope bc, increase slightly upon addition of A. herba Alba oil, which signify that cathodic sites are blocked by the adsorption of the molecules resulting in an inhibition of the cathodic reduction reactions. Cathodic Tafel lines were extrapolated to the corrosion potential so as to establish polarization settings got for aluminum corrosion in $\mathrm{HCl}\left(1 \mathrm{~mol} . \mathrm{L}^{-1}\right)$ solution without and in the presence of diverse concentrations of A. herba-alba oil. Alike fitting method had been 
previously used for aluminum in hydrogen chloride solution (Şafak et al.,2012) Corrosion potentials ( $\mathrm{E}_{\text {corr }}$ ), corrosion current densities (Icorr), cathodic Tafel slope values (bc) deducted from the polarization curves, matching inhibition efficacies $(\eta(\%))$ and surface coverage degrees $(\theta)$ are presented in Table 4.

The displacement of corrosion potentials in the cathodic meaning and diminution of corrosion currents with the augmentation of inhibitor concentration is a clue that the molecules of the cathode site are adsorbed on the surface of the aluminum (Bereket et al., 2004). By addition of A. herba-alba oil to the $\mathrm{HCl}$ solution, an upturn in corrosion conduct is evident. This could be chiefly ascribed to the physical adsorption process of active molecules, which besides form a protective layer on aluminum surface.

Table 4 Potentiodynamic polarization settings recorded for aluminium in $\mathrm{HCl}$ solution $1 \mathrm{M}$ with and without $A$. herba-alba oil at $303^{\circ} \mathrm{K}$

\begin{tabular}{lccccc}
\hline $\begin{array}{l}\mathbf{C}_{\text {inh }} \\
(\mathbf{g} / \mathbf{L})\end{array}$ & $\begin{array}{c}\mathbf{E}_{\text {corr }} \\
(\mathbf{m V / A g A g C l})\end{array}$ & $\begin{array}{c}-\mathbf{b}_{\mathbf{c}} \\
(\mathbf{m V / d e c})\end{array}$ & $\begin{array}{c}\mathbf{I}_{\text {corr }} \\
\left(\mathbf{m A . c m} \mathbf{A n}^{-2}\right)\end{array}$ & $\boldsymbol{\theta}$ & $\boldsymbol{\eta}^{\prime} \mathbf{( \% )}$ \\
\hline 0 & -755 & 155.0 & 3.20 & - & - \\
0.2 & -770 & 183.9 & 0.80 & 0.75 & 75.0 \\
0.5 & -774 & 190.0 & 0.25 & 0.92 & 92.1 \\
0.8 & -780 & 198.0 & 0.16 & 0.95 & 95.0 \\
\hline
\end{tabular}

\section{Electrochemical impedance spectroscopy (EIS)}

The EIS spectra of an aluminum electrode, in a molar solution of hydrochloric acid in the absence and presence of different $A$. herba alba oil concentrations, plotted at OCP, after 1 hour of stabilizing in solution ventilated at $303^{\circ} \mathrm{K}$, are shown in Fig 5. The Nyquist plots display ulike form composed of depressed capacitive loop in the high frequency (HF) region followed by an inductive loop in the low frequency (LF) region. The HF capacitive loop is assigned to the charge transfer process of the electric double layer at the electrode/electrolyte interface. As can be seen, lower diameters of capacitive and inductive loops are observed in blank solution, which suggest higher charge transfer and higher deterioration of the protective film of corrosion products. The LF inductive loop translated an adsorption and pitting corrosion phenomenon owing to the presence of chloride ions when the electrode undergo active dissolution followed by the destruction of the passive film (Metikoš-Huković et al., 1994). Moreover, with addition of A. herba-alba oil in acidic solution, remarkable increase in impedance magnitude is noticed. This indicates that with the rise of the inhibitor concentration, charge transfer process and pit propagation mechanism are impeded.

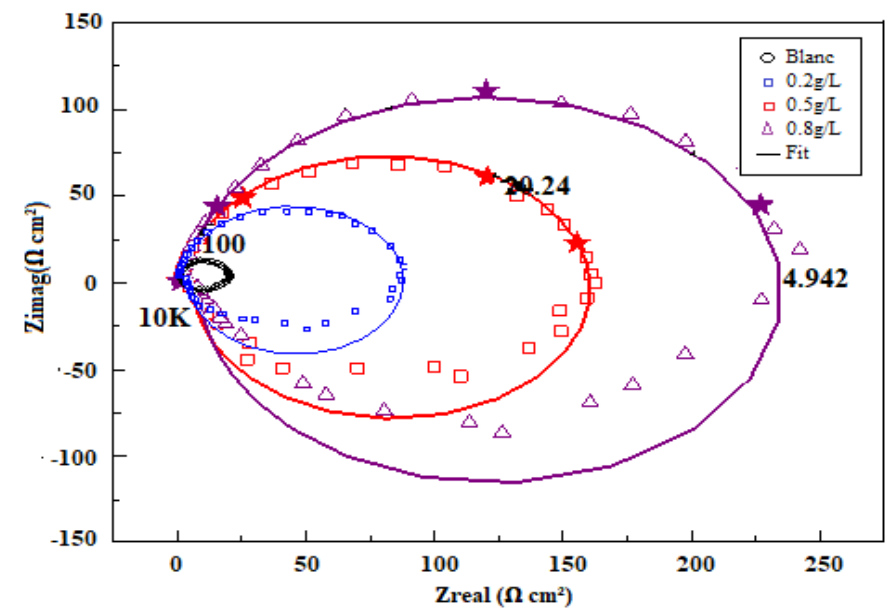

Figure 5 Nyquist plot for Aluminum in $\mathrm{HCl}(1 \mathrm{~mol} . / \mathrm{L})$ solution with and without $A$. herba-alba oil after $1 \mathrm{~h}$ at $\mathrm{OCP} \mathrm{T}=303 \mathrm{~K}$

The equivalent circuit model (ECM) shown in Fig 6, was proposed in order to permit a precise analysis of the diagrams of impedance. The electrical elements corresponding to the dynamic processes can be defined as follows:

Rs is the resistance of the solution, CPE represent the constant phase element and $\mathrm{RP}$ is the polarization resistance. Inductive elements $\left(\mathrm{R}_{\text {ind }}\right.$ and $\left.\mathrm{L}\right)$ are quite common for aluminum impedance behavior in acidic solution (Metikoš-Huković et al., 1994). The sum of $R P$ and $R_{\text {ind }}$ represents the charge transfer resistance $R_{c t}$. CPE was used in lieu of an ideal capacitor, since capacitive loops are not perfect circles, and this can be correlated to the frequency dispersion as a result of the in homogeneity and ruggedness of electrode surface (Khaled et al., 2009).

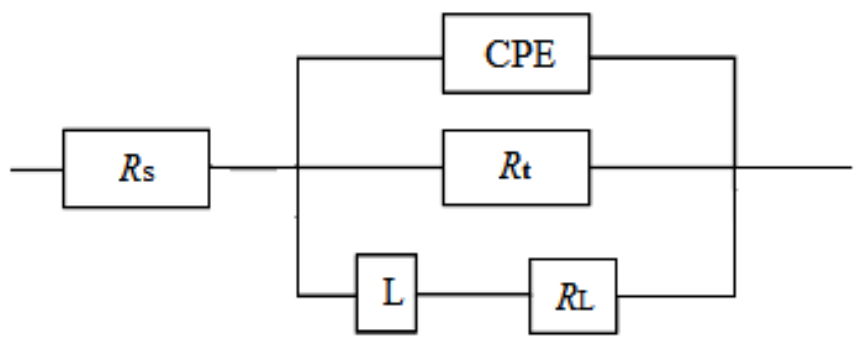

Figure 6 Equivalent circuit used to adjust impedance data

The impedance settings deduced from Nyquist plots obtained for aluminum in hydrochloric acid (1 mol./L) and the impedance parameters and inhibition efficacies $\eta$ eis $(\%)$ for the addition of essential oil in $\mathrm{HCl}$ solution, are presented in Table 5

Table 5 Characteristic settings deducted from Nyquist plot

\begin{tabular}{|c|c|c|c|c|c|c|c|c|c|}
\hline $\begin{array}{l}\text { C } \\
(\mathrm{g} / \mathrm{L})\end{array}$ & $\begin{array}{c}R s \\
\left(\Omega \mathrm{cm}^{2}\right)\end{array}$ & $\begin{array}{c}R p \\
\left(\Omega \mathbf{c m}^{2}\right)\end{array}$ & $\begin{array}{c}\mathbf{f} \max \\
(\mathbf{H z})\end{array}$ & $\begin{array}{c}\mathrm{CPE} \\
\left(\mu \mathrm{F} / \mathrm{cm}^{2}\right)\end{array}$ & $\mathbf{n}$ & $\begin{array}{c}R_{\text {ind }} \\
\left(\Omega \mathrm{cm}^{2}\right)\end{array}$ & $\begin{array}{c}\mathrm{L} \\
\left(\mathrm{H} \mathrm{\textrm {cm } ^ { 2 }}\right)\end{array}$ & $\begin{array}{c}\mathbf{R}_{\mathrm{ct}} \\
\left(\Omega \mathrm{cm}^{2}\right)\end{array}$ & $\eta(\%)$ \\
\hline 0 & 1.20 & 1.94 & 602.10 & 62.1 & 0.9609 & 2.25 & 7.68 & 14.25 & - \\
\hline 0.2 & 1.18 & 2.86 & 147.70 & 53.3 & 0.9435 & 3.00 & 17.68 & 62.00 & 77.0 \\
\hline 0.5 & 1.50 & 5.79 & 92.49 & 24.8 & 0.9366 & 6.00 & 36.31 & 165.00 & 91.4 \\
\hline 0.8 & 1.25 & 8.68 & 57.90 & 6.3 & 0.9299 & 8.00 & 53.98 & 247.00 & 94.3 \\
\hline
\end{tabular}

The results reveal that $R_{c t}$ values rise significantly, whereas CPE decreases with the augmentation of the concentration of A. herba-alba oil. This diminution of $\mathrm{CPE}$ values compared to that in the solution of $\mathrm{HCl}$ not containing essential oil can arise from an increase in the thickness of the electrical double layer and suggest that molecules present in A. herba alba oil are obstructing the metal surface by their adsorption at the interface of the metal/solution (Singh et al., 2011). The highest inhibitor efficiency $(94.3 \%)$ has been recorded in the presence of $0.8 \mathrm{~g} / \mathrm{L}$ of $A$. herba-alba oil. This result is close with that obtained from potentiodynamic polarization method. A similar study reported by Hechiche et al., (2019) showed an inhibitory efficacy more or less low (92\% for a concentration of $3 \mathrm{~g} / \mathrm{l})$ in comparison to our results. This may be due to the difference in chemical composition of the essential oil of $A$. herba alba, knowing that the major compound reported in this study is camphor $26.2 \%$ and chrysanthenone $12.4 \%$ while our study presents chrysanthenone $24.1 \%$ and camphor $16.2 \%$ as the major compounds.

\section{Gravimetric measurement}

The effect of Artemisia herba-alba oil concentration on the corrosion of $\mathrm{Al}$ in 1 $\mathrm{mol} / \mathrm{L} \mathrm{HCl}$ solution was examined by weight loss measurements at $303 \mathrm{~K}$ after an immersion of $1 \mathrm{~h}$. Table 6 exhibits the calculated values of corrosion rates, surface coverage and inhibition effectiveness in the absence and presence of various concentrations of Artemisia oil. As can be seen, corrosion rate decreases with the increase concentration of A. herba-alba oil. This decrease of "v" values in comparison with that in $\mathrm{HCl}$ solution without essential oil can result mainly from the formation of a protective film originated by the strong adsorption of the inhibitor molecules at the metal/solution interface (Metikoš-Huković et al., 1994). The inhibitory effect of Artemisia herba-alba essential oil is superior at higher concentration; the maximum of $94.3 \%$ is attained at $0.8 \mathrm{~g} / \mathrm{L}$. These results agree well with those recorded from potentiodynamic polarization method.

Table 6 Weight loss measurements after $1 \mathrm{~h}$ at $303^{\circ} \mathrm{K}$

\begin{tabular}{|c|c|c|c|c|}
\hline$C(g / L)$ & $\Delta \mathrm{m}$ (g) & $\begin{array}{c}\mathrm{v}\left(\mathrm{mg} \cdot \mathrm{cm}^{-2} \cdot \mathrm{h}^{-}\right. \\
1)\end{array}$ & $\eta{ }^{\prime \prime}(\%)$ & $\boldsymbol{\theta}$ \\
\hline 0 & 0.157 & 14.0 & - & - \\
\hline 0.2 & 0.040 & 3.50 & 75.0 & 0.75 \\
\hline 0.5 & 0.017 & 1.53 & 89.1 & 0.89 \\
\hline 0.8 & 0.010 & 0.81 & 94.3 & 0.94 \\
\hline
\end{tabular}




\section{CONCLUSIONS}

The analysis of essential oil isolated from A. herba-alba plant shows that it composition is dominated by Ketones $(61.1 \%)$. The major constituents are chrysanthenone (24.1\%), camphor (16.2\%) and $\alpha$-thujone (12.8\%). The essential oil showed a good activity against all microorganisms tested (MIC not exceeding 2 $\mu \mathrm{g} / \mathrm{mL}$ ) except for Aspergillus ochraceus where the MIC is $5 \mu \mathrm{g} / \mathrm{mL}$. Bacillus coagulans, Staphylococcus aureus, Agrobacterium tumefaciens, Fusarium oxysporum, Penicillium expansum and Pseudomonas aeruginosa were the most sensitive microorganisms tested (MIC $0.5 \mu \mathrm{g} / \mathrm{mL}$ ). The essential oil was found to be less active by inhibiting free radicals compared to the synthetic antioxidants (BHT and BHA). Maximum value of inhibition efficiency $(94.3 \%)$ was reached at $0.8 \mathrm{~g} / \mathrm{L}$. SEM studies revealed improvements in Al surface in presence of A. herba-alba essential oil which confirms the formation of an adsorbed and protective barrier of A. herba-alba essential oil molecules. The polarization studies showed that A. herbaalba essential oil acts as mixed-type inhibitor without changing the hydrogen evolution mechanism. From EIS measurement it is clear that the charge tra a perfect correlation between the magnitude of the inductive loop and the propagation of pitting corrosion. Good agreement was obtained between the different methods employed in this study and showed that $A$ herba-alba can be a good candidate for aluminium corrosion protection in hydrochloric acid medium.A transfer resistance increase with the inhibitor concentration and charge transfer process and pit propagation mechanism are impeded. SEM analysis allows

According to these findings, this essential oil may be suggested as new green inhibitor agent against corrosion in hydrochloric acid media and the possible use of the essential oil on food systems as an effective inhibitor of foodborne pathogens and for potential pharmaceutical applications. However, further research is needed in order to determine the toxicity, antibacterial, and antioxidant effects in edible products.

Acknowledgments: We are grateful the Professor Azziz Kadri of Materials Physics and Chemistry Laboratory of University Mouloud Mammeri for his support of the present survey.

\section{REFERENCES}

Adams, R.P. (2001). Identification des composants de l'huile essentielle par chromatographie en phase gazeuse/spectrométrie de masse quadripolaire. $2 \mathrm{e}$ éd. Séduit Publ. Corp.; Carol Stream, Illinois : États-Unis. Google Scholar

Akrout, A. (2004). Etude des huiles essentielles de quelques plantes pastorales de la région de Matmata (Tunisie). Cah Options Med, 289-292. http://www.ciheam.org/04600172

Ating, A.I., Umoren, S.A., Udousoro, I.I., Ebenso, E.E., Udoh, A.P. (2010) Extrait de feuilles d' Ananas sativum comme inhibiteur de corrosion verte pou l'aluminium dans des solutions d'acide chlorhydrique Green Chem. Lett. Review, (3) 2 : 61-68. https://doi.org/10.1080/17518250903505253.

Andreani, S., Znini, M., Paolini, J., Majidi, L., Hammouti,B., Costa, J., Muselli, A. (2016). Study of Corrosion Inhibition for Mild Steel in Hydrochloric Acid Solution by Limbarda crithmoides (L.) Essential Oil of Corsica. J mater Environ Sci, 7: 187-195. http://www.jmaterenvironsci.com/Document/vol7.

Bailey, C., Danin, A. (1981). Bedouin plant utilization in Sinai and the Negev.Econ. Bot, 35 (2):145-162. http://www.jstor.org/stable/42542722.

Benjilali, B., Richard, H. (1980). Etude de quelques peuplements d'Armoise blanche du Maroc Artemisia herba-alba. Riv. Ital. EPPOS, 62, 69-74 http://plantearomatiquemedicinale

Benabdellah, M., Benkaddour, M., Hammouti, B., Bendahhou, M., Aouniti, A. (2006). Inhibition of steel corrosion in 2M H3PO4 by Artemisia oil. Appl. Surf. Sci, 252 (18) : 6212-6217. https://doi.org/10.1016/j.apsusc.2005.08.0300.

Benjilali, B. ; Sarris, J. ; Richard, H. (1981). Nouveaux chemotypes d'Artemisia herba-alba. Sciences des Aliments, $2: 515-527$.

Bereket, G., Pinarbas, A. (2004). Electrochemical thermodynamic and kinetic studies of the behaviour of aluminum in hydrochloric acid containing various benzotriazole derivatives. Corros. Eng. Sci. Technol, 39: 308 https://doi.org/10.1179/174327804X131366.

Bezza, L., Mannarino, A., Fattarsi, K., Mikail, C., Abou, L., Hadji-Monaglou, F., Kaloustian, J. (2010). Composition chimique de l'huile essentielle d'Artemisia herba alba provenant de la région de Biskra (Algérie), Phytothérapie, 8 : 277-281 https://doi.org/10.1007/s10298-010-0576-3

Boudalia, M., Fernández-Domene, R.M., Tabyaoui, M., Bellaouchou, A., Guenbour, A., García-Antón' J.. (2019). Green approach to corrosion inhibition of stainless steel in phosphoric acid of Artemesia herba albamedium using plant extract, Journal of Materials Research and Technology, 8(6): 5763-5773. https://doi.org/10.1016/j.jmrt.2019.09.045.

Boutekdjiret, C., Cherchari, S., Belabbes, R., Bessiere, J. M. (1992). Contribution à l'étude de la composition chimique de l'huile essentielle d'Artemisia herba-alba, Riv. Ital. EPPOS, 3: 39-42.

Bouyanzer, A., Hammouti, B. (2004). A study of anti-corrosive effects of Artemisia oil on steel. Resin \& Pigment Technol, 33 (5): 287-292. http://www.jmaterenvironsci.com.
Brand-Williams, W., Cuvelier, M. E., Berset, C. (1995). Use of free radical method to evaluate antioxidant activity. Lebensm.-Wiss.-Technol, 28: 25-30 https://doi.org/10.1016/S0023-6438(95)80008-55.

Branzoi, V., Golgovici, F., Branzoi, F. (2003). Aluminium corrosion in hydrochloric acid solutions and the effect of some organic inhibitors. Materials Chemistry and Physics,78(1): 122-131. https://doi.org/10.1016/S0254 0584(02)00222-5

Chauhan, L.R., Gunasekaran, G. (2007). Corrosion inhibition of mild steel by plant extract in dilute $\mathrm{HCl}$ medium. Corros. Sci, 49: 1143-1161. http://dx.doi.org/10.1016/j.corsci.2006.08.0122

Dahmani N (2010). Etude de la composition chimique de l'Artemisia herba-alba poussant en Algérie. Thèse de doctorat en chimie, Univ USTHB, Algérie.

Dahmani-Hamzaoui, N., Baaliouamer, A. (2010). Chemical composition of Algerian Artemisia herba-alba essential oils isolated by microwave and hydrodistillation. J. Essent. Oil Res, 22: 514-517. https://doi.org/10.1080/10412905.2010.97003866

Dahmani-Hamzaoui, N., Baaliouamer, A. (2015). Volatile constituents of Algerian Artemisia herba-alba essential oils. J. Essent. Oil Res, 10: 37-41. https://doi.org/10.1080/10412905.2015.10274188

Dahmani-Hamzaoui, N., Sabaou, N., Salido, S., Altarejos, J., Baaliouamer, A (2018). Composition, antibacterial and antioxidant activities of Artemisia herba alba essential oils from Algeria. J. Fundam. Appl. Sci, 10(3): 13-32. https://dx.doi.org/10.4314/jfas.v10i3.2

Dahmani-Hamzaoui, N., Salido, S., Linares-Palomino, P., Baaliouamerc, A., Altarejos, J. (2012). On-Line Radical Scavenging Detection and Characterization of Antioxidants from Artemisia herba-alba. Helvetica Chimica Acta, 95: 564 576. https://doi.org/10.1002/hlca.201100367

Dob, T., Benabdelkader, T. (2006). Chemical composition of the essential oil of Artemisia herba-alba Asso grown in Algeria. J. Essent. Oil Res, 18: 685-690. https://doi.org/10.1080/10412905.2006.96992066

Feuerstein, L., Danin, A., Segal, R. (1988). Constitution of the essential oil from Artemisia herba-alba population of Spain. Phytochemistry, 27: 433-434. https://doi.org/10.1016/0031-9422(88)83114-55

Giordani, R., Hadef, Y., Kaloustian, J. (2008). Compositions and anti-fungal activities of essential oils of some Algerian aromatics plantes. Fitoterapia, 79: 199 211. https://doi.org/10.1016/j.fitote.2007.11.0044

Hachelef, H., Benmoussat, A., Khelifa, A., Athmani, D., Bouchareb, D. (2016) Study of corrosion inhibition by Electrochemical Impedance Spectroscopy method of 5083 aluminum alloy in $1 \mathrm{M} \mathrm{HCl}$ solution containing propolis extract. J. Mater Environ. Sci, 7 (5): 1751-1758. https://www.jmaterenvironsci.com.

Hadjadj, N., Hazzit, M. (2020). Analysis and antioxidant activity of essential oils and methanol extracts of Origanum floribundum Munby. J Essent Oil-Bear Plants, 23:85-96. https://doi.org/10.1080/0972060X.2020.1729867.

Halambek, J., Žutinić, A., Berković, K. (2013). Ocimum basilicum L. Oil as Corrosion Inhibitor for Aluminium in Hydrochloric Acid Solution. Int. $J$. Electrochem. $\quad \mathrm{Sci}$ 8: http://www.electrochemsci.org/papers/vol8/80911201.pdf.

Haouari, M., Ferchichi, A. (2009). Essential oil composition of Artemisia herbaalba from southern Tunisia. Molecules, 14: 1585-1594. https://doi.org/10.3390/molecules140415855.

Hazzit, M., Baaliouamer, A., Veríssimo, A.R., Faleiro, M.L., Miguel, M.G. (2009). Chemical composition and biological activities of Algerian Thymus oils Food Chem, 116:714-721. https://doi.org/10.1016/j.foodchem.2009.03.0188 Hechiche, N., Boughrara, D., Kadri, A., Dahmani, N., Benbrahim, N. (2019). Artemisia herba alba essential oil as green corrosion inhibitor for aluminum in hydrochloric acid solution. Anal. Bioanal. Electrochem, 11(8): 1129-1145. https://hal.archives-ouvertes.fr/hal-02428588 Hudaib, M., Aburjai, T. (2003) Composition of the essential oil from Artemisia herba-alba grown in Jordan, $J$ Essen. Oil Res. 18 : 301-304. https://doi.org/10.1080/10412905.2006.9699096 Hussin, M. H., Kassim, M. J., Razali, N.N. (2016). The effect of Tinospora crispa extracts as a natural mild steel corrosion inhibitor in $1 \mathrm{M} \mathrm{HCl}$ solution. Arabian Journal of Chemistry, 9: S616-S624. https://doi.org/10.1016/j.arabjc.2011.07.002 Jouad, H., Haloui, M., Rhiouani, H., Hilaly, J.E., Eddouks, M. (2001) Ethnobotanical survey of medicinl plants used for the treatment of diabetes, cardiac and renal diseases in the North centre region of Morocco (Fez-Boulemane). $J$ Ethnopharmacol, $77 \quad$ (2-3): 175-182. https://doi.org/10.1016/S0378 8741(01)00289-6

Khadraoui, A., Khelifa, A. (2013). Ethanolic extract of Ruta chalepensis as an ecofriendly inhibitor of acid corrosion of steel. Res. Chem. Intermed, 39: 3937-3948 https://doi.org/10.1007/s11164-012-0910-5.

Khadraoui, A., Khelifa, A., Hachama, K. (2016). Thymus Algeriensis extract as a new eco-friendly corrosion inhibitor for 2024 aluminium alloy in $1 \mathrm{M} \mathrm{HCl}$ medium. Journal of Molecular Liquids, 214: 293 297. https://doi.org/10.1016/j.molliq.2015.12.064

Khaled, K. F., Al-Qahtani, M.M. (2009). The inhibitive effect of some tetrazole derivatives towards Al corrosion in acid solution: Chemical, electrochemical and theoretical studies. Mater. Chem. Phys, 113: 150-158. https://doi.org/10.1016/j.matchemphys.2008.07.060

Lakehal, S., Chaouia C., Benrebiha, F.Z. (2017). Chemical composition and antibacterial activity of the essential oil of Artemisia herba-aba Asso from djelfa Revue Agrobiologia, 7(2): 491-501. 
Mahmoud, S.A.S., Hazem, A., EL-Sebaie, M.A.F., El-Borollosy, M.A., EL-Dady, A.H. (1988). Microbiological studies on the phyllosphere of the desert plant $A$. herba-alba. Egyptian Journal of Microbiology, 22 (1): 55-77.

Masada, Y. (1979). Analysis of essential oils by GC/MS. John Wiley;New York. Metikoš-Huković, M., Babić, R., Grubac, Z, Brinć, S. (1994). Impedance spectroscopic study of aluminum and Al-alloys in acid solution : inhibitory action of nitrogen containing compounds. J. Appl. Electrochem, 24: 772-778. https://doi.org/10.1007/BF00578093

Mighri, H., Hajlaoui, H., Akrout, A., Najjaa, H., Neffati' M. (2010). Antimicrobial and antioxidant activities of Artemisia herba-alba essential oil cultivated in Tunisian arid zone. Comptes Rendus Chimie, 13 (3): 380-386. https://doi.org/10.1016/j.crci.2009.09.0088

Mohamed, A. E.-H. H., El-Sayed, M. A., Hegazy, M. E., Helaly, S. E., Esmail, A. M., Mohamed, N. S. (2010). Chemical Constituents and Biological Activities of Artemisia herba-alba. Rec. Nat. Prod, 4(1): 1-25.

Ouachikh, O., Bouyanzer, A., Bouklah, M., Desjobert, J.M., Costa, J., Hammouti, B., Majidi, L. (2009). Application of Essential Oil of Artemisia herba alba as green corrosion inhibitor for steel in 0.5M H2SO4. Surface Review and Letters, 16(1) 49-54. https://doi.org/10.1142/S0218625X09012287

Oussalah, M., Caillet, S., Sucier, L., Lacroix, M. (2007). Inhibitory effects of selected plant essential oils on the growth of four pathogenic bacteria: E. coli O157:H7, Salmonella Typhimurium, Staphylococcus aureus and Listeria $\begin{array}{llll}\text { monocytogenes. } & \text { Food } & \text { Control, } & \text { 18: }\end{array}$ https://doi.org/10.1016/j.foodcont.2005.11.009

Paolini, J., El Ouariachi, EM., Bouyanzer, A., Hammouti, B., Desjobert, J.M., Costa, J., Muselli, A. (2010). Chemical variability of Artemisia herba-alba Asso essential oils from east Morocco. Chemical papers, 64 (5): 550-556. https://doi.org/10.2478/s11696-010-0051-5

Salhi, A., Bouyanzer, A., Chetouani,A., El Barkany, S., Amhamdi, H., Hamdani, I., Zarrouk, A., Hammouti, B., Desjobert, J.M., Costa, J. (2017). Chemical composition of essential oil and antioxidant and anti-corrosion activity of extract and essential oil of Pennyroyal Mint (Mentha pulegium, MP), Mor. J. Chem, 5(1) 59-71. https://doi.org/10.48317/IMIST.PRSM/morjchem-v5i1.6788

Salido, S., Valenzuela, L. R., Altarejos, J. (2004). Composition and infraspecie variability of Artemesia herba-alba form southern spain. Biochem Syst Ecology, 13: 221-224. https://doi:10.1016/j.bse.2003.09.002

Safak, S., Duran, B., Yurt, A., Türkoğlu, G. (2012). Schiff bases as corrosion inhibitor for aluminum in $\mathrm{HCl}$ solution. Corros. Sci, 54: 251-259. https://doi.org/10.1016/j.corsci.2011.09.026

Segal, R., Feuerstein, L., Danin, A. (1987). Chemotypes of Artemisia herba alba based on their sesquiterpene lactone and essential oil constitution. Biochem. Syst. Ecol, 15: 411-416. https://doi.org/10.1016/0305-1978(87)90054-8

Singh, A., Ahamad, I., Singh, V.K., Quraishi, M.A. (2011). Inhibition effect of environmentally benign Karanj (Pongamia pinnata) seed extract on corrosion of mild steel in hydrochloric acid solution. J. Solid. State Electrochem, 15: 10871097. https://doi.org/10.1007/s10008-010-1172

Solmaz, R., Kardas, G., Ulha, M.C., Yazıcı, B., Erbil, M. (2008). Investigation of adsorption and inhibitive effect of 2-mercaptothiazoline on corrosion of mild steel in hydrochloric acid media, Electrochim. Acta, 53: 5941-5952. https://doi.org/10.1016/j.electacta.2008.03.055

Vagionas, K., Graikou, K., Ngassapa, O., Runyoro, D., Rhinou, I. (2007). Composition and antimicrobial activity of the essential oils of three Satureja species growing in Tanzania. Food Chemistry, 103: 319-324. https://doi.org/10.1016/j.foodchem.2006.07.051

Valnet, J. (1984). Aromatherapie: Traitement des Maladies par les Essences des Plantes, Maloine Editions, Paris

Vernin, G., Merad, L.O. (1994). Mass spectra and Kovats indices of some new cischrysanthenyl esters found in the essential oil of Artemisia herba-alba from $\begin{array}{lllll}\text { Algeria. } J . & \text { Essent. Oil Res, 6: 437-448 }\end{array}$ https://doi.org/10.1080/10412905.1994.9698419

Vernin, G., Merad, L.O., Vernin, G. M. F., Zamkotsian, R. M., Parkanyi, C. (1995) GC/MS analysis of Artemisia herba-alba Asso essential oils from Algeria. Dev. Food Science, 37(A): 147-205.

Vernin, G.; Parkanyi, C. (2001). GC/MS analysis of Artemisia herba-alba Asso from Algeria: non polar and polar extracts. Riv. Ital. EPPOS. 2001, 32, 3-16.

Yan, Y., Li, W., Cai, L., Hou, B. (2008). Electrochemical and quantum chemica study of purines as corrosion inhibitors for mild steel in $1 \mathrm{M} \mathrm{HCl}$ solution, Electrochim. Acta, 53,5953-5960 https://doi.org/10.1016/j.electacta.2008.03.065 Zouari S., Zouari N., Fakhfakh N., Bougatef A., Aydi M.A., Neffatil M. (2010). Chemical composition and biological activities of a new essential oil chémotype of Tunisian Artemisia herba alba Asso. Journal of Medicinal Plants Research, 4 871- 880. http://doi.org/ 10.5897/JMPR09 